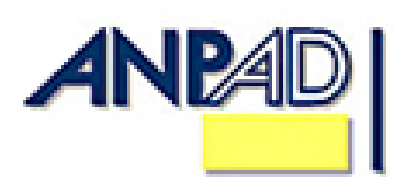

Available online at http://www.anpad.org.br/bar

BAR, Rio de Janeiro, v. 9, n. 1, art. 6, pp. 110-126, Jan./Mar. 2012

\title{
New Technologies, Old Habits: Automation without Innovation
}

José Osvaldo De Sordi *

E-mail address: de.sordi@ terra.com.br Universidade Nove de Julho - UNINOVE

São Caetano do Sul, SP, Brazil.

Manuel Meireles

E-mail address: profmeireles@uol.com.br Faculdade Campo Limpo Paulista - FACCAMP

Campo Limpo Paulista, SP, Brazil.

Marta Lígia Pomim Valentim

E-mail address: valentim@marilia.unesp.br

Universidade Estadual Paulista - UNESP São Paulo, SP, Brazil.

* Corresponding author: José Osvaldo De Sordi

Rua Iwakumi, 236, Jundiaí, SP, 13211-424, Brazil.

Copyright (C) 2012 Brazilian Administration Review. All rights reserved, including rights for translation. Parts of this work may be quoted without prior knowledge on the condition that the source is identified. 


\begin{abstract}
This research investigated the underuse of technological tools by innovative organizations which are acknowledged for their use of and familiarity with new technologies. The research conducted an analysis of 58 institutional repositories (IRs) out of 43 educational and research institutions which are internationally renowned for excellence. The core aspect of the analysis was the use of IRs for publishing and dealing with evidence in order to legitimize and add value to scientific research. The following items were analyzed: (a) the logical structuring of scientific communication published in the IRs; (b) the metadata which describe scientific communication based on the terms of the DCMI protocol used; (c) the availability of software functions which facilitate the queries and publication of evidences. Results show that the introduction of IRs did not add value to the quality of research in terms of associating and publishing evidence that could back them up. A strong tendency to replicate the traditional library model of physical collections was observed. It was concluded that merely possessing good technological tools is not sufficient for fostering innovation and strategic gains in organizations, even if their implementation takes place in highly promising and favorable environments.
\end{abstract}

Key words: institutional repository; research evidence; research communication; DSpace. 


\section{Introduction}

During the last few years much there has been much discussion in the academic and organizational environments about the strategic importance of information technologies (IT) as applied to business, and much discussion has been fomented by the publication of an article by Nicholas Carr (2003): IT doesn't matter. Carr described a change in the purchasing process of IT resources by the organizations. Prices used to be too high and currently there are many outlets for their purchase and acquisition. Carr stated that, from the moment computers and software became commonly available, they turned into commodities, which therefore diminished their strategic value.

A competitive advantage sustained exclusively by the possession of IT resources becomes evermore uncommon, scarce and fleeting, since competing companies now only need to acquire identical or similar technological resources.

More sustainable competitive advantages, with greater strategic value to the organizations, are no longer achieved by only having new IT resources, as they may be easily acquired and equalled by competitors, according to Carr. However, this does not mean that IT does not contribute strategically to an organization, as proposed by Carr. Peter Keen, for example, argued against the perception of IT as a resource fully destitute of strategic value (Keen as cited in Devaraj \& Kohli, 2002, p. 20): "When all companies inherently have access to the same information technology resources, the competitive differential and the economic benefits will depend on the IT management and not on technological differences".

This research deals with the implementation of IT resources in organizations, based upon the assumption that an information system may provide very different results, from several small gains obtained through automation to innovations that directly contribute to the main activity and competitive capability of an organization. As an example, even though the adoption of high quality, technologically mature tools has the potential to foster product and service innovations, they can be underutilized and provide only smaller gains due to automation (Hambrose, 2009; Keen, 1991). This concept served as impetus for this research, which was established by the following question: Can the underutilization of innovative technological tools occur even in institutions having a significant level of international excellence and that are operating in business segments with a good tradition in knowledge creation and innovation?

The objective of this research is to collect and analyze scientific research communication items published at institutional repositories (IR) at main educational and research institutions. The IRs are defined as information systems designed for storing, preserving, organizing, managing and disseminating the digital assets of organizations (Smith, 2002), thus, they constitute an essential tool for educational and research institutions. A significant share of the results obtained by these organizations derives from the publication of new knowledge generated by the scientific research produced within the institutions.

Various free, open source software and specific software titles are available for developing IRs: DSpace (http://www.dspace.org), EPrints (http://www.eprints.org), Fedora (http://www.fedoracommons.org), among others. This research deals with the DSpace system due to its wide adoption by educational and research institutions. It was developed by the Massachusetts Institute of Technology (MIT) in partnership with Hewlett-Packard (HP); DSpace has been developed in Java for the Linux platform.

The objective of the research is to analyze the usage level of the resources available at the DSpace IRs by educational and research institutions which are internationally acknowledged for excellence. Among the multiple possible angles for analysis, priority has been given to the innovation characterized by the friendly and easy publication of the evidence (digital objects) which support the scientific research developed by educational and research institutions. The actual assumption is that 
any research which is published along with its evidence has a greater value by allowing readers to have a greater capacity for analyzing and perceiving its scientific value (Creswell, 2003).

Effectively relating and publishing evidence associated with the respective scientific research is one way of achieving an innovative use of IR and adding value to research institutions, since this is not something easy to implement in the physical world of libraries, and also because it creates direct gains for its main product: scientific research. Adding value takes place through the gains obtained from information quality. More specifically, evidence contributes in two qualitative dimensions of information, one related to the intrinsic nature of information and the other in a contextual manner with respect to its accuracy and completeness (Lee, Strong, Kahn, \& Wang, 2002).

Traditionally with paper media or even digital media without the use of IRs, evidence, when available, is part of the main file used for communication and dissemination of the scientific research. One example is evidence published in appendices at the end of textual documents in digital media (.DOC or .PDF). This type of evidence is not the focus of this research, since it does not benefit from the relational facilities available in database managing systems used in the technological architecture of IRs.

Database managing systems allow for the implementation of a conceptual model of entityrelationship, which is outstanding, as it manages to express real world situations with ease, simplicity and meaningful semantics. According to Chen (1977), the vision of a given reality is based upon the relationships among entities, as they portray the facts that define said reality, and that each one (entity or relation) may have qualifying attributes of the reality. The abstraction concept allows the analyst to distinguish and separate the parts which are really relevant to the development of the information system from all aspects which do not have any influence on the environment to be modeled.

It is therefore noted that at the core of this research is the analysis of relationships among entities and the logical abstractions obtained from them. The two groups of entities covered by this research are: (a) the ones composed by documents which divulge and characterize the research of the institutions, such as articles and theses, and (b) the ones associated with the evidence that support such research, such as transcriptions of interviews, calculations, spreadsheets, videos, photos and other related documents. The relationships between these two groups were analyzed with reference to how they are associated and represented in the DSpace IRs of the educational and research institutions.

This research investigated the existence of relationships between the two groups of entities: communication of scientific research, such as theses and articles, and the availability of research evidence associated with them. It is worth emphasizing that research evidence in digital format, which can be stored in the IR, is becoming more and more common, due to the process of product and services digitalization available in modern society. The most typical example for this process are the photo, video and audio records, which are generated in digital format and can be stored in the IRs. In short, the possibility of the existence of evidence in digital format associated with scientific communications is increasingly improving.

\section{Research of Quality: New Technologies and Publication of Evidences}

In science, it is essential to publish research evidence in order to assess its quality. The quality of research generally involves associative and comparative studies (triangulations) in many aspects: data and evidence collected in the field (data triangulation); viewpoints about the same set of data (triangulation of theory); and research techniques and methods (methodological triangulation) (Yin, 1994).

In academic communication, particularly through articles, researchers are unable to present the entire volume of information processed during the research, as scientific communication is necessarily concise. A typical example of this limitation is the production of articles that use Case Study strategy. 
Such articles require intense triangulation of data, including: transcriptions of dialogs which have taken place in interviews and/or focus groups, photos and videos of related entities, data collected through questionnaires, related tables, etc.

Multiple data sources promoting a more complete analysis are demanded due to the evolution of research methodologies, especially for qualitative research. Researchers are spending more and more effort associating different communication media (texts, sounds, photos, videos, graphics) which are transmitted and stored in different technological formats (analog, digital and physical). In order to help researchers handle the diversity and complexity imposed by data triangulation, specific software has been developed - the so-called Computer-Assisted Qualitative Date Analysis Software (Maclaran, $\&$ Catterall, 2002).

The good practices of scientific research dictate that not only scientific articles or scientific research reports should be published, but also all other material which is important for understanding the interpretations and analyses developed by the researcher, that is, the necessity for publication of the various sources of evidence which support the conducted analyses. This implies providing readers with access to the sources used: texts from transcriptions, videos, photos, calculation spreadsheets and other sources used in the triangulation efforts carried out by the researcher (Yin, 1994).

The concept of evidence has become highly important in different areas, such as medicine (Evidence-Based Medicine [EBM]) and information science (Evidence-Based Librarianship [EBL]). The organization of Library Science aims to improve the professional practice in the information sciences by means of evidence produced through investigation.

By giving priority to higher levels of evidence (that is, those using more quantitative methods) in determining the best available evidence for answering a particular question, EBL pursues the dual goals of encouraging research that exhibits both methodological rigor and relevance to practical situations in librarianship (Lerdal, 2006, p. 34).

The principle of this wide dissemination means allowing other researchers to review the evidence directly, without being limited to the restricted contents of reports, articles and other means of access to scientific research. This principle is associated with a group of factors that economists identify as the driving force of the human capability: a more in-depth concept of capital (labor force ability to use new equipment which is more productive than previous versions); greater work quality (better informed labor force, capable of adding value to economic results); and technological innovation - labor force ability to create, distribute, share and utilize new knowledge (Stiglitz, \& Walsh, 2002).

IR is one of the technological resources that help educational and research institutions meet new demands related to the publication of academic and scientific production. Developed with new resources of information technology, IRs provide: friendly graphical interfaces, search engines, ability to store entities in different formats (text, image, video, software, audio), among other facilities that help the activities of those who publish and search the collections resulting from research. Lynch (2003, p. 2) states that:

a university-based institutional repository is a set of services offered by the university to the members of the its community for the management and dissemination of digital materials created by the institution and its community members. It is most essentially an organizational commitment to the stewardship of these digital materials, including long-term preservation where appropriate, as well as organization and access or distribution.

In addition to the demand from the academic-scientific environment, the impetus for proliferation of IRs stemmed from technological breakthroughs, such as the reduction in the cost of electronic storage, the expansion of the Internet and the evolution of open communication protocols such as the Open Archives Initiative - Protocol for Metadata Harvesting (OAI-PMH). 
Through sharing metadata, facilitated by the OAI-PMH model, authors and editors who are interested in making their publications available need not add new services to their information products. This is one of the several ways universities provide for the dissemination of the results of scientific research, by creating mechanisms to legitimate and encourage the publication of studies (Lynch, 2003).

Lomangino (2006, p. 2) points out some benefits to institutions brought by IR:

Repositories can therefore be justified based on the increased grant support that they may be able to help generate for the institution. Researchers and faculty are also expected to benefit from the increased visibility associated with repositories. Since repositories are typically defined as open access systems, the content that resides there should, in theory, receive more use from the academic community because it is free. This may translate into higher citation rates than comparable material published in subscription-only journals. Moreover, repositories remove what many academics consider the artificial space limitations of printed journals, allowing for more and different kinds of information to be published. As these constraints are lifted, researchers can expect more of their own work and that of colleagues to become available for review.

Within academic institutions, the bond between the Library (Document Management) and the IRs seems undeniable:

In the long-term, organizing and maintaining digital content-as well as supporting faculty as information contributors and end users - should remain the responsibility of the library. Libraries are best suited to provide much of the document preparation expertise ... to help authors contribute their research to the institution's repository. Similarly, libraries can most effectively provide much of the expertise in terms of metadata tagging, authority controls, and the other content management requirements that increase access to, and the usability of, the data itself (Crow, 2007, p. 20).

Establishing an IR indicates that the library is changing its custody role in order to actively contribute actively to the evolution of the scientific communication model. Libraries keep their responsibility of managing and filing printed materials, but as the volume of material for open access research in digital format grows, the role and the value of the printed collections shall lessen proportionally. Because libraries are better prepared to provide the normalization for preparation of digital documents, they help the work of authors and contribute to their research in IRs (Pinfield, Gardner, \& Macoll, 2002).

The Dublin Core Metadata Initiative (DCMI) is one of efforts organized to provide interoperability of data between creators and users. Even so, within this program, there are optional terms which aim at providing the creator and publisher of contents the ability to customize the description process. DCMI is an international standard for describing information resources, and it is considered an important part of the Internet infrastructure. It was first developed in 1994 by a group of librarians and specialists in contents, led by Stuart Weibel, researcher of the Online Computer Library Center (OCLC). It consists of a set of 15 (fifteen) elements of metadata, equivalent to a card catalog, which may be considered the least common denominator for describing information resources (Weibel, 1997).

DSpace only accepts the Dublin Core standard for describing the contents of the items stored in it, although there is research in progress for developing components that allow the usage of random metadata projects using the Resource Description Framework (RDF). DSpace makes available the 15 Dublin Core basic elements adopted individually or in association with 46 qualifiers, which have been developed based on the Dublin Core Libraries Working Group Application Profile (LAP).

The syntactic and semantic structure of the DCMI protocol is adequate for describing IR entities that relate to each other, regardless of their types and formats. When defining the adherence to the DCMI protocol, DSpace benefits from the intellectual assets associated with it, in other words, 
facilitating the publication of, and the access to, scientific collections at institutions. In this scenario of protocol adoption, DSpace is equipped with a network tool focused on the public outside the institution. The further the IR reading public is from the information generating source, the greater the need for explicitness and transparency of the information published in the IR, that is, the greater the need for publication and association of evidence.

Effectively relating and publishing evidence associated with respective research is one way of achieving an innovative use of IR and adding value to research institutions, since this is not something easy to implement in the physical world of libraries, and also because it creates direct gains for its main product: the scientific research. Table 1 exhibits some important associations among entities relevant to IRs that may be established with the use of the terms isPartOf and hasPart of the DCMI protocol.

Table 1

Examples of Relationships among Institutions Stored in the IR

\begin{tabular}{ll}
\hline Entity & IsPartOf \\
\hline Article & conference annals, seminar, symposium, magazine \\
book chapter & book \\
evidence of research & communication of research \\
\hline Entity & HasPart \\
\hline Conference & article, summary \\
book & book chapter \\
scientific magazine & article, review \\
communication of research & research evidence \\
\hline
\end{tabular}

Note. Source: developed by the authors.

DCMI ( 2009 p. 4) defines the term dc.format as "the format of the file, physical media or the dimensions of the resource", whereas the term dc.type is described as "the nature or genre of the resource" (p. 6). The term dc.format can be correctly defined by the information center analyst only by conducting a superficial analysis of the entity to be registered in the IR, that is, it depends exclusively upon the analysis of external aspects of the physical or logic characteristics of the information. It is about a process which requires little involvement between the analyst and the informational entity. It is possible, for example, to define the Text/HTML format for a certain entity without having to open the file for an analysis of its contents: it suffices to identify the file extension of the object which is being registered. On the other hand, the definition of the string to be defined for the term dc.type requires a greater involvement by the information center analyst with the entity to be registered in the IR: the text file needs to be opened, regardless of its format being .DOC or .PDF, in order to ascertain the nature of the information, whether it is an article, a thesis, a working paper or any other type of document.

\section{Method}

An exploratory research confronted the top 100 institutions considered among the best educational and research institutions that use the DSpace system (available in http://www.dspace.org/whos-using-dspace/Repository-List.html). Forty-three of these institutions were identified as users of the DSpace IR system, some of them having more than one DSpace IR implemented, resulting in a total of 58 DSpace IR to be researched. 
The names of the host institution, the country where they are located, and the Internet address (URL) of each one of 58 DSpace IRs that constituted the sample of the research are described in Appendix. Data gathering for this research took place between July and December 2009. The following items were observed: (a) the logical structuring of scientific communication published in the IRs; (b) the metadata which describe scientific communication based on the terms of the DCMI protocol used; (c) the availability of software functions which facilitate the querying activities and the publication of evidence. The following search procedures were adopted for the analysis of the DSpace IRs:

a) For the basic search option, thesis was entered; for the thesis list generated, five records were selected randomly and they were handled in the following fashion:

- The presentation of all the descriptive attributes of the document was requested, and therefore, the show full item record option was selected;

- The use of the terms isPartOf and hasPart of the protocol DCMI was analyzed for the description of the record;

- The number of files attached to the record was checked, as well as their type (conceptual nature) and their format (electronic media).

b) For the basic search option, article was entered;

For the generated list of articles, five records were selected randomly which were treated with the same procedures and analyses used for thesis.

c) For the advanced search option, one verified the possibility of conducting a search on the documents stored in the DSpace IR according to the following criteria:

. Format of the stored documents, the term format of the DCMI (dc.format) and

. Type of the documents stored, the term type of the DCMI (dc.type).

From these three search procedures it was observed that 50 of 58 IRs DSpace published research results in the format of thesis, dissertation, article or working paper. The other eight IRs are from institutions that possess more than one RI, some which are quite specific in orientation. As example, New York University possesses a RI - Digital Afghanistan Library - specifically addressed to publication of digital files of books, pamphlets and manuals published in Afghanistan during the period of 1871-1930.

\section{Analysis of publication of evidence for scientific research available in the DSpace IRs}

In most of the IRs researched, the records related to scientific communication of the institutions are published in text format using one of the two following technologies: (a) Microsoft Word standard files (.DOC.); or (b) portable document format for different platforms developed by Adobe Systems (.PDF). The text of scientific communication can be found in a single file or broken down into multiple files, in which case they are often all compressed into a single file by means of data compression software, such as a Zip File (.ZIP). An example of scientific communication broken down into multiple files is the theses made available by the Australian National University IR. Most of them are broken down into two files: one of them containing only pre-text elements (cover, summary, lists of figures and tables) and the other containing the body of thesis text itself. Other IRs publish each chapter of the scientific communication, such as a thesis or a research report, in an individual file; however, they are all stored and made available as a single record of the IR.

In the IRs researched, the occurrence of publications of research communication, as well as its evidence, was not found by using more than one record associated through terms of DCMI defined for this purpose, such as: isPartOf, hasPart and relation. The little evidence of scientific research found 
in the IRs that were separated from the main body of the scientific communication were mostly contained in the same record as that of the research communication, and structured by the application of two technical procedures: (a) scientific communication files and evidence files published jointly in the same record, that is, one record of the IR containing several attached files; (b) scientific communication text files and evidence files in the same record, compressed in a single zip file (.ZIP). An example of the first procedure can be seen in the dataset record of Richardson (2009) located in the IR called DataShare of the University of Edinburgh. In this same university, however, in another IR Edinburgh Research Archive - the master dissertation record of Buchanan (2007) includes evidence in a compressed file (.ZIP).

It should be emphasized that the publication of compressed files (.ZIP) in the IRs is not an advised practice, because it corrupts the principles of atomicity and uniqueness required by relational and normalized environments (respect to first normal form - 1FN - of the Entity-Relationship Model (Chen, 1977)). The Internet Assigned Numbers Authority is the institution in charge of the supervision of the list of strings for the description of application types (MIME Type or Content-type). Although this institution shows the ZIP string in its list, it does not recommend its use due to an intrinsic inconsistency of its essence with regards the accuracy required by communication protocols.

During the research, the use of the terms isPartOf and hasPart was observed for other purposes other than the association between evidence and research. At the Ohio State University IR, the composition of two DC terms is utilized - relation and isPartOf - to associate articles with the conferences where they were presented. For the essay of Judkins (2009) recorded in the IUScholarWorks Repository the term dc.relation.ispartofseries was used. Similarly, Indiana University at Bloomington used such terms to associate articles with the scientific journals that published them, as is observed in the book reviewed record of Sobecki (2009). The record of Green, Lorenzoni, Perez and Pollitt (2006) found in the IR of the University of Cambridge, associates a working paper with a collection of assets of the institution: dc.relation.ispartofseries was used. In the IR of The Ohio State University, the doctoral dissertation record of Ryba (2009) uses the following terms to indicate the department of the University in charge of the thesis: "dc.relation.ispartofseries: The Ohio State University. Department of Arts Honors Theses".

For the few situations identified which were related to publication of evidence and research communication in different records within the same DSpace IR, it was observed that the logic association between them was established through the use of inappropriate DCMI terms, that is, the failing to use the terms isPartOf, hasPart and relation. For example, the DCMI terms title (dc.title) and summary (dc.abstract) of a record being used in an improper way. The dataset record of Day (2008) found in the IR of the University of Cambridge exhibits the results of a calculation whose logic is described in a thesis. The association between the results and the thesis that describes the logic of the calculation occurs by means of the term dc.description.abstract. The most appropriate DCMI term for this declaration would be isPartOf, which would indicate the address or title of another IR record containing said thesis. Such an approach would associate the record of a scientific communication, in this particular case the thesis, with the research evidence and calculations performed.

Another distortion of the use of the DC protocol caused by the failure to use proper terms for the description of relationships between parts (isPartOf, hasPart and relation) was observed in the DSpace IR of the Massachusetts Institute of Technology. The software files record of Thomson (2008) describes a software title which is evidence for a thesis, and is also stored in the same DSpace IR. The association between both occurs by means of the name of the record which describes the software: "dc.title: Software files to support thesis of Ty Thomson" (Thomson, 2008, p. 1).

The IRs managers have shown little concern in valuing the comprehension of "the nature or genre of the resources" stored, described by dc.type of DCMI (2009, p. 6). In DCMI, the standardized description with the highest level of information essence associated with a record occurs by means of the term dc.type. Table 2 describes the IRs which allow searches of stored documents by type, and it is worth noting that only 7 of the 58 IRs researched (12\%) enable searches by the term dc.type. 
Among those, two show a set of possible values with characteristic problems due to the lack of precision and formality in the management of valid words for the term dc.type, for instance characterized by the repetition of words according to the first letter of the word, in either lowercase or uppercase (image and Image; Video and video), or according to the word being singular or plural (Article and Articles; Report and Reports). Redundancy may also occur due to typos (Corresondence, Corresponcence, Correspondence and Correspondence).

Table 2

\section{Description of the IRs which Allow Searching by the Term Type (dc.type) of the Stored Documents}

Rice University, RUDR URL: http://dspace.rice.edu/

There is a page for specifically searching by TYPE with $\mathbf{8 1}$ of possible types of documents to be searched: Albums; Application forms; Architectural drawing; ;Architectural drawings; Article; Articles; Book; Book Chapter; Brochures; Business cards; Card photographs; Christmas cards; Clippings; Conference Paper; Construction drawings; Contracts; Corresondence; Corresponcence; correspondence; Correspondence; Dedications (Documents); Documents; Drafts (documents); Drawings; Elec 599 Project Report; Estimates; Exhibition catalogs; Frontispieces (illustrations); Illumination of books and manuscripts, European; Illumination of books and manuscripts, European; image; Image; Internal Document; Invention Report; Invitations; Invoices; Journal Paper; Land surveys; Lectures; Letter; letters (correspondence); Masters Thesis; Memorandums; Minutes; MS 352 Box 1 Folder 18 Item 18; MS Thesis; News clipping; Notes; Other; Pamphlets; PhD Thesis; Photographs; Photograph; Photographs; Picture postcard; Picture postcards; poetry; Progress schedules; Postcards; Presentation; Type; Programs; Report; Reports; Resume; Sales records; Sketchbooks; Sketches; Souvenir programs; Specifications; Speech; Still Image; Summaries; Tech Report; Technical Report; Telegram; Telegrams; Text; text; Thesis; White Paper; Writings.

Rice University, TIMEA URL: http://timea.rice.edu/

The advanced search page shows 4 possible types of documents to be searched: Images; Texts; Maps; Research Modules.

University of Cambridge URL: http://www.dspace.cam.ac.uk/

There is a page for a specific search by TYPE with 25 possible types of documents to be searched: Article; Audio; Book or Book chapter; Book or Book Chapter; BW Image; Colour; Image; Dataset; Drawn image; Image; image; Journal Article - Published Version; Journal Article - Submitted Version; Learning Object; Manuscript; Map; Other; Preprint; Presentation; Software; Table; Thesis; Video; video; webpage; Working Paper.

University of Illinois at Urbana-Champaign URL: http://www.ideals.uiuc.edu/dspace/

The advanced search page does not show a set of types to be defined (check list); however, there is an option for choosing search by TYPE and typing the string of characters corresponding to the name of the desired TYPE.

University of Zurich URL: http://www.zora.unizh.ch/zora/

The advanced search page shows 8 possible types of documents to be searched: Book Section; Conference or Workshop Item; Dissertation; Edited Scientific Work; Habilitation; Journal Article; Monograph; Newspaper Article.

The search may be refined through the selection of a sub-type: Original work (original research) or Further contribution (e.g. review article, editorial).

The advanced search page shows 8 possible types of documents to be searched: Book Section; Conference or Workshop Item; Dissertation; Edited Scientific Work; Habilitation; Journal Article; Monograph; Newspaper Article.

The search may be refined through the selection of a sub-type: Original work (original research) or Further contribution (e.g. review article, editorial). 


\section{Table 2 (continued)}

\begin{tabular}{|c|c|}
\hline $\begin{array}{l}\text { Universiteit Utrecht, } \\
\text { Igitur Archive }\end{array}$ & $\begin{array}{l}\text { URL: http://igitur- } \\
\text { archive.library.uu.nl/search/search.php?m=simple\&language=en\&p=1 }\end{array}$ \\
\hline \multicolumn{2}{|c|}{$\begin{array}{l}\text { The advanced search page shows } 8 \text { possible types of documents to be searched: Article; Book; Book review; } \\
\text { Dissertation; Lecture; Map; Preprint; Report. }\end{array}$} \\
\hline The Ohio State University & URL: https://kb.osu.edu/dspace/index.jsp \\
\hline
\end{tabular}

Note. Source: developed by the authors

The lack of precision in the use of the term dc.type by DSpace IR, as well as its scarce use as a search element of the collection, characterizes the continuity of the cultural tradition deriving from traditional libraries: not organizing and not searching by types of documents of the collection, instead taking into consideration the traditional ways and the strong predominance of contents such as books and magazines in traditional physical collections.

When analyzing the adoption of the format of the stored document, the term dc.format of DCMI as a search criteria in the DSpace IR collection was rarely observed, although to a somewhat greater extent than the term dc.type: Twelve out of the 58 IRs (21\%) allow searches according to the format of the media used, according to Table 3.

Table 3

Description of the IRs which Allow Searches by the Term Format (dc.format) of the Stored Documents

TECHNIQUE USED: On advanced search options, those allowing selection of <MIME Type> and typing the string of the term to be searched

\begin{tabular}{ll}
\hline Indiana University Bloomington & https://scholarworks.iu.edu/dspace/ \\
Johns Hopkins University & https://jhir.library.jhu.edu/ \\
Massachusetts Institute of Technology & http://dspace.mit.edu/ \\
Texas A\&M University, Geological Atlas of the & \\
United States & http://txspace.tamu.edu/handle/1969.1/2490 \\
Texas A\&M University, Institutional Repository & http://txspace.tamu.edu/ \\
University of Edinburgh, DataShare & http://datashare.edina.ac.uk/dspace/ \\
University of Helsinki, Viikki campus & http://www.tiedekirjasto.helsinki.fi/dspace/ \\
University of Toronto Library, ResearchSpace & https://researchspace.library.utoronto.ca/ \\
University of Washington, ResearchWorks & https://digital.lib.washington.edu/dspace/ \\
\hline
\end{tabular}

TECHNIQUE USED: On advanced search options, those allowing selection of <MIME Type> and typing the string of the term to be searched

\begin{tabular}{ll}
\hline $\begin{array}{l}\text { University of Wisconsin } \\
\text { Vanderbilt University }\end{array}$ & http://minds.wisconsin.edu/ \\
http://discoverarchive.vanderbilt.edu/
\end{tabular}

Note. Source: developed by the authors 
In the organizational environment, (libraries and information centers included) when analyzing the context of the informational environment, a greater domain and perception of the aspects and characteristics related to materiality and to physical or logic composition of the information and the documents as related to digital documents were observed. The understanding of the materiality of the information, whether in physical or virtual format, predominates over its own essence, while it represents a communication and semantic value, according to Davenport's (1997, p. 146) comment about information users in the context of the organizations: "Ask them what information they use and need, and they'll be speechless. However, they know the documents they use, enjoy and value".

\section{Conclusions}

Based on the information assessed from the 58 DSpace IRs researched, it can be stated that there are important and intrinsic potentialities for the storage technology of relational contents, which have been little explored by the 43 institutions that manage these environments. Few declared that relationships have been identified among the internal records of a given IR that used more adequate DC terms, such as: isPartOf, hasPart and relation. For the association between scientific research communication and its evidence, in lieu of these terms, other, less adequate terms for DC were used, such as: dc.title and dc.abstract.

Most of the research evidence found in the DSpace IRs analyzed is still maintained in the old traditional library format and its physical collection in paper format: they are all inserted in the same scientific research document. The only difference is that it is in a digital media format such as .DOC. or .PDF instead of paper media. Due to this, only minor gains from the introduction of new technologies (automation) are obtained, such as faster access to, and faster search of, the collection. For the cases of individual records storing the communication of research and its evidence with an improper use of the DC protocol terms, it is unfeasible to take advantage of the facilities of abstraction and logic association among institutions, which are available in relational technologies. An example of functional limitations due to the inadequate use of the technological infrastructure available in the DSpace IR is the impossibility of using it as search criteria, or simply to inform the users who do scientific research in these databases, about the quantity and the types of evidence associated with theses, articles and other forms of scientific communication available.

The DSpace IRs analyzed showed little availability of research resources by type of document stored - it was found in only 7 out of the 58 IRs researched (12\%). This is an indicator of little positive action for publication of evidence, which is normally available in different types of documents other than articles, theses or research reports, but instead consisting of: photos, software, videos, texts, maps, among other (strings) considered document types (dc.type). For those documents which are not characterized as scientific communication, their existence in terms of media (dc.format) is also different from the usual text format used in articles, theses, research reports and other types of scientific communication. A video-type document may be implemented in different formats, such as $\mathrm{mp} 4$, vnd, 3gpp or mpv. Twelve out of the investigated 58 DSpace IR (21\%) allow searches of their records to be made according to the media format (dc.format). This result shows little interest by the institutions towards the diversity of technological formats of their contents, which is an intrinsic aspect of the environments which do not prioritize the publication of evidence deriving from scientific research.

With respect to the scientific information quality published by the educational and research institutions, more specifically about the validity of the information published, it can be stated that the introduction of the DSpace IR technology has a potential to add value to institutions, for example, through an easy and practical method of displaying evidence associated with scientific communication. From this viewpoint, it can also be stated that there is a potential for a greater valorization and strategic use of the 58 analyzed DSpace IRs. 
The current stage of the environments analyzed is that of technological introduction, considering the short usage time of the DSpace IR technology itself: which became available for use in September 2002 (Swartz, 2003). The history of the introduction of new information technology resources in organizational environments indicates that it will be necessary to invest more time and effort in order to obtain a greater added value for the educational and research institutions, as far as DSpace IR environments are concerned. This may involve a change in the behavior of librarians and researchers at institutions, so that there can be an evolution from the current stage of the traditional physical model of the library to a virtual environment endowing better performance. The prevailing practice nowadays is the publication of the same existing structure of scientific communication found in traditional libraries, (i.e. long texts in a single document) although no longer in paper format, but in digital format. In short, there has been little gain from the automation related to the introduction of new information technologies, which could provide greater availability of the information and greater speed for retrieving and searching such information.

The organizational environments involved in the conducted analyses were very promising in terms of fostering the adoption of the new technology, by creating results which were meaningful to organizations. In other words, they should go beyond the traditional gains obtained through automation. The introduced technology - DSpace - was developed by two institutions which complement one another very well in terms of a demand needing to be met: The Massachusetts Institute of Technology (MIT), with strong knowledge and tradition in scientific publication and in development of technologies related to information, and Hewlett-Packard (HP), with a solid tradition in the development of solutions based on information technology resources and in converting and applying innovations to organizational contexts. In addition to the credibility and tradition of the institutions which have developed the technology analyzed, it is worth mentioning the similarity in terms of research excellence, innovation, tradition and international credibility of the 43 institutions which use the DSpace IR solution and which constituted the sample population of this research.

Despite the fact that the environments involved in this research are favorable to innovative adoption of new technologies, this has not actually occurred. This helped provide an answer to the main objective of this research: making good technological tools available is not enough in itself to promote innovation and provide strategic gains to organizations, even when such resources are made available in highly favorable environments having a tradition in innovation and in the creation and adoption of new technologies. The scope of this research did not cover the discussion and consideration of the causes for such a conclusion. Rather, it analyzed the explicitness and evidence of the phenomenon.

In this research, the analysis of the potential aspects of the DSpace IR associated with the evidence as connected to scientific communication was given priority, since it constituted a significant resource for adding value to organizations that were using this technology: the educational and research institutions. A great deal of the perception of quality and success of these institutions is linked to the perception of their research quality, expressed in the form of scientific communication, which strongly depends upon readers' analyses of the degree of accuracy, honesty, and validity of the information published. This, in turn, is directly associated with the availability and transparency of evidence substantiating the research which has been carried out.

As a follow-up to the current research, an investigation could be enacted jointly with the research institutions that intensively use DSpace technology for the implementation of their IRs, as to the causes for not associating and not publishing research evidence. With this as a goal, different research fronts can be created, considering that there are many dimensions begging to be investigated, such as cultural, behavioral, technological, and ethical dimensions, among others.

\section{Received 4 May 2010; received in revised form 16 September 2010.}




\section{References}

Buchanan, C. (2007). Techniques for solving nonlinear programming problems with emphasis on interior point methods and optimal control problems (Doctoral dissertation). Department of Mathematics and Statistics, University of Edinburgh, Edinburgh, Scotland. Retrieved September 17, 2009, from http://hdl.handle.net/1842/2387

Carr, N. G. (2003). IT doesn't matter. Harvard Business Review, 81(5), 41-49.

Chen, P. P. (1977). Data base management: the entity-relationship approach to logical database design. Wellesley, MA: Q.E.D. Information Sciences, Inc.

Creswell, J. W. (2003). Research design: qualitative, quantitative, and mixed methods approaches. Thousand Oaks, CA: Sage Publications, Inc.

Crow, R. (2007). The case for institutional repositories: a SPARC position paper. Washington: The Scholarly Publishing and Academic Resources Coalition. Retrieved August 22, 2009, from http://www.arl.org/sparc/repositories/readings.html

Davenport, T. H. (1997). Information ecology: mastering the information and knowledge environment. New York: Oxford University Press, Inc.

Day, N. E. (2008). Results files for organic solid-state PM6 calculations from Nick Day's PhD thesis. [Data file]. Department of Chemistry. University of Cambridge, Cambridge, England. Retrieved September 15, 2009, from http://www.dspace.cam.ac.uk/handle/1810/197582

Dublin Core Metadata Initiative. (2009). Dublin Core Metadata Element Set, Version 1.1. Retrieved September 21, 2009, from http://dublincore.org/documents/dces/

Devaraj, S., \& Kohli, R. (2002). The it payoff: measuring the business value of information technology investments. New Jersey: Prentice Hall.

Green, R. J., Lorenzoni, A., Perez, Y., \& Pollitt, M. G. (2006). Benchmarking electricity liberalisation in Europe [Working paper]. Faculty of Economics, University of Cambridge, UK. Retrieved September 19, 2009, from http://www.dspace.cam.ac.uk/handle/1810/131686

Hambrose, H. (2009). Wrench in the system: what's sabotaging your business software and how you can release the power to innovate. New York: Wiley.

Judkins, R. (2009). Who will break the deer? Lord and huntsman in medieval english hunting ritual. Proceedings of the Hayes Graduate Research Forum, Columbus, OH, USA, 23. Retrieved September 16, 2009, from http://hdl.handle.net/1811/37026

Keen, P. (1991). Every manager's guide to information technology. Boston: Harvard Business School Press.

Lee, Y. W., Strong, D. M., Kahn, B. K., \& Wang, R.Y. (2002). AIMQ: a methodology for information quality assessment. Information \& Management, 40(2), 133-146. doi: 10.1016/S03787206(02)00043-5

Lerdal, S. N. (2006). Evidence-based librarianship: opportunity for law librarians? Law Library Journal, 98(1), 33-60.

Lomangino, K. (2006). Institutional repositories: their emergence and impact on scholarly publishing. Pennsylvania: The Sheridan Press. 
Lynch, C. A. (2003). Institutional repositories: essential infrastructure for scholarship in the digital age. Association of Research Libraries, (226), 1-7. Retrieved from http://www.arl.org/resources/pubs/br/br226/br226ir.shtml

Maclaran, P., \& Catterall, M. (2002). Analysing qualitative data: computer software and the market research practitioner. Qualitative Market Research: An International Journal, 5(1), 28-39. doi: $10.1108 / 13522750210414490$

Pinfield, S., Gardner, M., \& Macoll, J. (2002). Setting up an institutional e-print archive. Ariadne, 31. Retrieved from http://www.ariadne.ac.uk/issue31/eprint-archives/intro.html

Richardson, E. A. (2009). Carstairs deprivation scores by CATT2, 1981, 1991, 2001 [Data file]. Retrieved September 15, 2009, from http://hdl.handle.net/10283/19

Ryba, C. R. (2009). Scandinavian functionalist ceramics \& the landscape (Doctoral dissertation). Department of Art Honors, The Ohio State University, Columbus, OH, USA. Retrieved September 10, 2009, from http://hdl.handle.net/1811/37006

Smith, M. (2002). DSpace: An open source institutional repository for digital material. D-Lib Magazine, 8(10). Retrieved from http://www.dlib.org/dlib/october02/10inbrief.html

Sobecki, S. (2009, November 24). Ward, world of the medieval shipmaster reviewed by Sobecki. The Medieval Review. Retrieved December 17, 2009, from http://hdl.handle.net/2022/6608

Stiglitz, J., \& Walsh, C. (2002). Principals of macroeconomics (3rd ed.). New York: Norton.

Swartz, N. (2003). MIT's super archive. Information Management Journal, 37(2), 18.

Thomson, T. (2008). Software files to support thesis of Ty Thomson. [Software]. Cambridge, MA: Author. Retrieved September 18, 2009, from http://hdl.handle.net/1721.1/41841

Weibel, S. (1997). The dublin core: a simple content description model for electronic resources. Bulletin of the American Society for Information Science, 24(1), 9-11. doi: 10.1002/bult.70

Yin, R. K. (1994). Case study research: design and methods (2nd ed.). Thousand Oaks, CA: Sage Publications, Inc. 


\section{APPENDIX}

Table 1

\section{Institutional Repositories (IRs) that Composed the Research Sample}

\begin{tabular}{|c|c|c|}
\hline Learning institution in charge of DSpace IR & Country & Internet address (URL) \\
\hline Australian National University & Australia & 1. http://dspace.anu.edu.au/ \\
\hline California Institute of Technology/NASA & USA & \\
\hline Jet Propulsion Laboratory & & 2. http://trs-new.jpl.nasa.gov/dspace/ \\
\hline Columbia University & Canada & 3. http://academiccommons.columbia.edu/ \\
\hline Cornell University & USA & 4. http://dspace.library.cornell.edu/ \\
\hline Duke University & USA & \\
\hline DukeSpace & & 5. http://dukespace.lib.duke.edu/ \\
\hline Medical Center Library and Archives & & 6. http://dspace.mclibrary.duke.edu/ \\
\hline The Duke Student Portfolio & & 7. https://portfolio.oit.duke.edu/index.jsp \\
\hline Harvard University & USA & 8. http://repository.countway.harvard.edu \\
\hline Imperial College London & UK & 9. http://spiral.imperial.ac.uk/ \\
\hline Indiana University Bloomington & USA & 10. https://scholarworks.iu.edu/dspace/ \\
\hline Johns Hopkins University & USA & 11. https://jhir.library.jhu.edu/ \\
\hline Kyoto University & Japan & 12. http://repository.kulib.kyoto-u.ac.jp/dspace/ \\
\hline Massachusetts Institute of Technology & USA & 13. http://dspace.mit.edu/ \\
\hline New York University & USA & \\
\hline Afghanistan Digital Library & & 13. http://afghanistandl.nyu.edu/ \\
\hline Archive@NYU & & 14. http://dspace.nyu.edu/ \\
\hline Modiya Project & & 15. http://modiya.nyu.edu/ \\
\hline North-West University & USA & 16. http://dspace.nwu.ac.za/ \\
\hline Rice University & USA & \\
\hline RUDR & & 17. http://dspace.rice.edu/ \\
\hline TIMEA & & 18. http://timea.rice.edu/ \\
\hline Texas A\&M University & USA & \\
\hline Geological Atlas of the United States & & 19. http://txspace.tamu.edu/handle/1969.1/2490 \\
\hline Institutional Repository & & 20. http://txspace.tamu.edu/ \\
\hline The Ohio State University & USA & 21. https://kb.osu.edu/dspace/index.jsp \\
\hline Tohoku University & Japan & 22. https://ir.library.tohoku.ac.jp/re/ \\
\hline Universität Göttingen & Germany & 23.http://geoleoedocs.sub.unigoettingen.de:8080/dspace/ \\
\hline Universiteit Leiden & Netherlands & 24. http://openaccess.leidenuniv.nl/dspace/ \\
\hline Universiteit Utrecht & Netherlands & \\
\hline Deposit Only & & 25. http://dspace.library.uu.nl:8080/index.jsp \\
\hline Igitur Archive & & $\begin{array}{l}\text { 26.http://igiturarchive.library.uu.nl/search/search.php?m=sim } \\
\text { ple\&language=en } \& \mathrm{p}=1\end{array}$ \\
\hline University of Arizona & USA & 27. https://dlearn.arizona.edu/ \\
\hline
\end{tabular}


Table 1 (continued)

\begin{tabular}{|c|c|c|}
\hline Learning institution in charge of DSpace IR & Country & Internet address (URL) \\
\hline University of Bristol & UK & 28. http://rose.bris.ac.uk/dspace/ \\
\hline University of British Columbia & USA & 29. https://circle.ubc.ca/ \\
\hline University of Cambridge & UK & \\
\hline DSpace Cambridge & & 30. http://www.dspace.cam.ac.uk/ \\
\hline Computer Lab Tech Reports & & 31. http://www.cl.cam.ac.uk/TechReports/ \\
\hline University of Edinburgh & UK & \\
\hline DataShare & & 32. http://datashare.edina.ac.uk/dspace/ \\
\hline EDINA & & 33. http://gradedemo.edina.ac.uk/dspace \\
\hline ERA & & 34. http://www.era.lib.ed.ac.uk \\
\hline University of Helsinki & Finland & \\
\hline Meilahti Campus & & 35. http://tds.terkko.helsinki.fi/ \\
\hline National Library of Finland & & 36. https://oa.doria.fi/ \\
\hline Viikki campus & & 37. http://www.tiedekirjasto.helsinki.fi/dspace/ \\
\hline University of Illinois at Urbana-Champaign & USA & 38. http://www.ideals.uiuc.edu/dspace/ \\
\hline University of Manchester & UK & 39. http://dspace.man.ac.uk:8080/dspace/ \\
\hline University of Maryland & USA & 40. https://drum.umd.edu/dspace/index.jsp \\
\hline University of Michigan, Ann Arbor & USA & 41. http://deepblue.lib.umich.edu/index.jsp \\
\hline University of Minnesota & USA & 42. http://conservancy.umn.edu/ \\
\hline University of North Carolina & USA & 43. http://etd.ils.unc.edu/dspace/ \\
\hline University of Oslo & Norway & \\
\hline Centre for European Studies & & 44. http://www.arena.uio.no/publications/ \\
\hline University of Pittsburgh & USA & 45. http://crystal.exp.sis.pitt.edu:8080/dspace/ \\
\hline University of Southern Califórnia & USA & 46. http://ir.usc.edu:8180/dspace/ \\
\hline University of Texas at Austin & USA & \\
\hline School of Information & & 47. https://pacer.ischool.utexas.edu/index.jsp \\
\hline University of Texas Libraries & & 48. http://repositories.lib.utexas.edu/ \\
\hline University of Tokyo & Japan & 49. http://repository.dl.itc.u-tokyo.ac.jp/dspace/ \\
\hline University of Toronto & Canada & \\
\hline ResearchSpace & & 51. https://researchspace.library.utoronto.ca/ \\
\hline Research Repository, T-Space & & 52. https://tspace.library.utoronto.ca/ \\
\hline University of Utah & USA & \\
\hline Utah Heavy Oil Center & & 53. http://ds.heavyoil.utah.edu/dspace/index.jsp \\
\hline University of Washington & USA & \\
\hline Health Sciences Libraries & & 54. http://dspace.hsl.washington.edu/dspace/ \\
\hline ResearchWorks & & 55. https://digital.lib.washington.edu/dspace/ \\
\hline University of Wisconsin & USA & 56. http://minds.wisconsin.edu/ \\
\hline University of Zurich & Switzerland & 57. http://www.zora.unizh.ch/zora/ \\
\hline Vanderbilt University & USA & 58. http://discoverarchive.vanderbilt.edu/ \\
\hline
\end{tabular}

